Louisiana State University

LSU Digital Commons

Faculty Publications

Department of Physics \& Astronomy

$12-10-2015$

\title{
NEW ULTRAVIOLET EXTINCTION CURVES for INTERSTELLAR DUST in M31
}

\author{
Geoffrey C. Clayton \\ Louisiana State University \\ Karl D. Gordon \\ Space Telescope Science Institute \\ Luciana C. Bianchi \\ Johns Hopkins University \\ Derck L. Massa \\ Space Science Institute \\ Edward L. Fitzpatrick \\ Villanova University
}

See next page for additional authors

Follow this and additional works at: https://digitalcommons.Isu.edu/physics_astronomy_pubs

\section{Recommended Citation}

Clayton, G., Gordon, K., Bianchi, L., Massa, D., Fitzpatrick, E., Bohlin, R., \& Wolff, M. (2015). NEW ULTRAVIOLET EXTINCTION CURVES for INTERSTELLAR DUST in M31. Astrophysical Journal, 815 (1) https://doi.org/10.1088/0004-637X/815/1/14

This Article is brought to you for free and open access by the Department of Physics \& Astronomy at LSU Digital Commons. It has been accepted for inclusion in Faculty Publications by an authorized administrator of LSU Digital Commons. For more information, please contact ir@lsu.edu. 
Authors

Geoffrey C. Clayton, Karl D. Gordon, Luciana C. Bianchi, Derck L. Massa, Edward L. Fitzpatrick, R. C. Bohlin, and Michael J. Wolff

This article is available at LSU Digital Commons: https://digitalcommons.Isu.edu/physics_astronomy_pubs/824 


\title{
NEW ULTRAVIOLET EXTINCTION CURVES FOR INTERSTELLAR DUST IN M31*
}

\author{
Geoffrey C. Clayton ${ }^{1}$, Karl D. Gordon ${ }^{2,3}$, Luciana C. Bianchi ${ }^{4}$, Derck L. Massa ${ }^{5}$, \\ Edward L. FitzPatrick ${ }^{6}$, R. C. Bohlin ${ }^{2}$, and Michael J. WOLFF ${ }^{5}$ \\ ${ }^{1}$ Department of Physics \& Astronomy, Louisiana State University, Baton Rouge, LA 70803, USA; gclayton@ fenway.phys.lsu.edu \\ ${ }^{2}$ Space Telescope Science Institute; 3700 San Martin Drive, Baltimore, MD 21218, USA; bohlin@stsci.edu, kgordon@stsci.edu \\ ${ }^{3}$ Sterrenkundig Observatorium, Universiteit Gent, Gent, Belgium \\ ${ }^{4}$ Department of Physics and Astronomy, The Johns Hopkins University, 3400 N. Charles Street, Baltimore, MD 21218, USA; bianchi@jhu.edu \\ ${ }^{5}$ Space Science Institute, 4750 Walnut Street, Suite 205, Boulder, CO 80301, USA; mjwolff@ spacescience.org \\ ${ }^{6}$ Department of Astronomy and Astrophysics, Villanova University, 800 Lancaster Avenue, Villanova, PA 19085, USA; edward.fitzpatrick@villanova.edu \\ Received 2015 April 17; accepted 2015 October 23; published 2015 December 2
}

\begin{abstract}
New low-resolution UV spectra of a sample of reddened OB stars in M31 were obtained with the Hubble Space Telescope/STIS to study the wavelength dependence of interstellar extinction and the nature of the underlying dust grain populations. Extinction curves were constructed for four reddened sightlines in M31 paired with closely matching stellar atmosphere models. The new curves have a much higher signal-to-noise ratio than previous studies. Direct measurements of $N(\mathrm{H}$ I $)$ were made using the Ly $\alpha$ absorption lines enabling gas-to-dust ratios to be calculated. The sightlines have a range in galactocentric distance of $5-14 \mathrm{kpc}$ and represent dust from regions of different metallicities and gas-to-dust ratios. The metallicities sampled range from solar to 1.5 solar. The measured curves show similarity to those seen in the Milky Way and the Large Magellanic Cloud. The Maximum Entropy Method was used to investigate the dust composition and size distribution for the sightlines observed in this program, finding that the extinction curves can be produced with the available carbon and silicon abundances if the metallicity is super-solar.
\end{abstract}

Key words: dust, extinction - ISM: abundances - ISM: molecules - ultraviolet: ISM

\section{INTRODUCTION}

Cardelli et al. (1989, CCM) found that the wavelength dependence of extinction in the Galaxy was a function of one parameter, $R_{V}\left(=A_{V} / E(B-V)\right)$, the ratio of total to selective extinction. This work was extended to over 400 sightlines in the Galaxy of which only four differed significantly from CCM (Valencic et al. 2004). However, it has become increasingly apparent that "standard" Milky-Way-(MW-) type dust extinction does not generally apply to interstellar dust in other galaxies. It has been known for a long time that the UV extinction properties in the Magellanic Clouds are different from the MW and from each other. In particular, sightlines near 30 Dor in the Large Magellanic Cloud (LMC; Clayton \& Martin 1985; Fitzpatrick 1985) and in the star-forming Bar of the Small Magellanic Cloud (SMC; Prevot et al. 1984) show very un-MW extinction

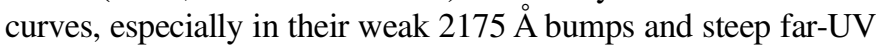
rises. The variations seen in dust properties extend beyond that of the extinction curve shape, including, e.g., the gas-to-dust ratio, indicating that there may be a wide range of underlying grain populations. In addition, many starburst galaxies seem to manifest dust properties similar to those associated with the SMC (Gordon \& Clayton 1998).

The CCM study only probed a limited set of dust environments in the MW. All of the sightlines were within $1 \mathrm{kpc}$ of the Sun, so it is not surprising that extinction in other galaxies has different properties than the MW. The observed variations from galaxy to galaxy are an indication of the complexity of dust properties and demonstrate the need for direct studies of dust for environments more representative of

\footnotetext{
* Based on observations made with the NASA/ESA Hubble Space Telescope, obtained by the Space Telescope Science Institute, and from the data archive at STScI. STScI is operated by the Association of Universities for Research in Astronomy, Inc., under NASA contract NAS5-26555.
}

the current sample of extragalactic spectral energy distributions (SEDs; e.g., Calzetti et al. 1994; Gordon et al. 1997).

The wavelength dependence of UV extinction due to dust can only be directly measured along sightlines where the spectra of individual stars can be obtained. Otherwise, the dust extinction characteristics must be inferred through radiative transfer modeling (Calzetti et al. 1994; Gordon et al. 1997). This technique is therefore limited to galaxies in the Local Group. Almost all of the studies of UV extinction have been limited to stars in three galaxies, the MW (Witt et al. 1984; Aiello et al. 1988; Fitzpatrick \& Massa 1990, 2007; Clayton et al. 2000; Valencic et al. 2004), the LMC (Clayton \& Martin 1985; Fitzpatrick 1985; Misselt et al. 1999; Gordon et al. 2003; De Marchi \& Panagia 2014, and the SMC (Lequeux et al. 1982; Prevot et al. 1984; Gordon \& Clayton 1998; Gordon et al. 2003, 2015; Maíz Apellániz \& Rubio 2012).

Beyond the MW and the Magellanic Clouds, there is not much information on the extinction properties in other Local Group galaxies. Radiative transfer modeling of the emission from the starburst nucleus of M33 indicates the presence of a strong $2175 \AA$ A bump unlike other starbursts, which show more SMC-like extinction having little or no evidence of a bump (Gordon et al. 1999). A recent Swift and Hubble Space Telescope (HST) photometric study of dust clumps in the bulge of M31 suggests that the UV extinction is generally steeper than the CCM, $R_{V}=3.1$ curve (Dong et al. 2014). Analysis of interstellar polarization along four sightlines in M31 suggests significant differences in the size distribution of silicate grains compared with those studied elsewhere in the Local Group (Clayton et al. 2004).

\section{DUST AND GAS IN M31}

Almost 20 years ago, Bianchi et al. (1996) did a "pilot study" using HST/FOS of UV extinction toward a very small 
sample of OB stars in M31. They derived an average M31 extinction curve from only three sightlines that has an overall wavelength dependence similar to that of the average Galactic extinction curve, but potentially possessing a weaker $2175 \AA$ bump. While the extinction curves calculated from these data provided a proof-of-concept, the study suffered several drawbacks that made interpretation of the extinction difficult: the UV spectra had a very low signal-to-noise ratio $(\mathrm{S} / \mathrm{N})$ and the stars observed were only lightly reddened.

The metallicity of M31 has been determined to be about two times solar by Zaritsky et al. (1994) based on H II regions. Studies of some A and B supergiants in M31 suggested that the metallicity was close to solar (Venn et al. 2000; Smartt et al. 2001), but a large, recent study by Sanders et al. (2012) has reconfirmed the super-solar abundances.

In this paper, we present new high-S/N HST/STIS spectra of a small number of significantly reddened stars in M31 and construct extinction curves for those sightlines to study the nature of its interstellar dust.

\section{OBSERVATIONS}

Eleven early-type M31 supergiants were observed by HST/ STIS using the G140L and G230L gratings. The sample was selected from stars known to be members of M31 for which spectral types are available (Massey et al. 1995; Bresolin et al. 2002; Cordiner et al. 2011). Two of the G140L observations, marked in Table 1 , failed and were redone. Also, three stars in the sample already had existing G140L observations. These were retrieved from the Mikulski Archive for Space Telescopes (MAST) and were used in this study. The new and archival observational data are summarized in Table 1. Table 2 lists the stellar parameters for the sample. The new UV spectra have been combined with existing ground-based UBVRI photometry (Massey et al. 2006) to create an SED for each star.

The sample of stars, for which new and archival HST/STIS spectra have been obtained, consists of seven significantly reddened stars $(E(B-V)=0.3-0.5)$ and four lightly reddened stars $(E(B-V) \sim 0.1)$ of similar spectral types. These stars are all members of M31 (Massey et al. 2006). The sample is limited to spectral types ranging from O7 to B2.5 supergiants (Massey et al. 1995; Bresolin et al. 2002; Cordiner et al. 2011).

Our most reddened star, J004412.17+413324.2, is very bright ( $V=17.33$ and $B-V=0.34$ Massey et al. 2006). If it is an early B supergiant, then the expected $M_{V} \sim-6.5$. With a calculated reddening $E(B-V)=0.49$ and assuming a distance modulus to M31 of 24.4 (e.g., Riess et al. 2012), then $M_{V}=$ $17.34-3.1 \times(0.49)-24.4=-8.6 \mathrm{mag}$. This is consistent with a bright blue supergiant and it has been confirmed as a member of M31 (Massey et al. 2006). Bonanos et al. (2003) found $\mathrm{J} 004412.17+413324.2$ to be a variable star.

Only one reddened star in our sample, J003944.71 +402056.2, has near-IR photometry $(J=17.83, K=17.68)$ (Sick et al. 2014). For this star, $E(V-K)=1.25$ assuming it is an $09.7 \mathrm{Ib}$ star (Cox 2000). Then, $A(V)$ can be estimated by $E$ $(V-K) \times 1.1=1.37 \mathrm{mag}$ (Whittet \& van Breda 1978), and $R_{V}=A(V) / E(B-V)=1.37 / 0.42=3.3$.

\section{EXTINCTION CURVES}

Traditionally, extinction curves are calculated using the standard pair method (Massa et al. 1983; Cardelli et al. 1992), which requires a reddened star and a lightly reddened comparison star having the same or similar spectral types. However, finding a good spectral match is not easy especially when a limited sample of lightly reddened stars is available, so stellar atmosphere models are now being extensively used as pair stars. The use of spectra from stellar atmosphere models as comparison "stars" is at least as accurate as using actual stellar spectra (Fitzpatrick \& Massa 2005). Extinction curves were attempted using the reddened and lightly reddened stars in the sample but the spectral type matches were not satisfactory. Extinction curve matches were made with stellar atmosphere models with better success.

The extinction curves were calculated by forward modeling of the spectrum of each star to determine the correct model atmosphere to use as the unreddened comparison star. The model we adopt for the M31 stars is a combination of a TLusty stellar model atmosphere (Lanz \& Hubeny 2003, 2007) extinguished by an $R(V)$-dependent extinction curve (Fitzpatrick 1999) in the optical and NIR, combined with a Fitzpatrick \& Massa (1990, FM) parametrization in the UV, updated by Fitzpatrick \& Massa (2007). The assumed model fit parameters are given in Table 3. Some of the model parameters are fixed at single values because our observations are not sensitive to these parameters. The M31 components of the model have radial velocities calculated using the method of Rubin \& Ford (1970) and Drout et al. (2009). These velocities are listed in Table 4. The MW components of the model have a fixed radial velocity of $0 \mathrm{~km} \mathrm{~s}^{-1}$.

We fit this model to the observed data using the EMCEE fitting code (Foreman-Mackey et al. 2013). The observed and model spectra were normalized by the average of the optical photometry prior to fitting. We imposed flat priors on most of the fit parameters with the min and max values given in Table 3. The min and max values were set to reasonable limits on fit parameters and were generally based on expected ranges from MW measurements of extinction curves (Valencic et al. 2004). The $\min$ and $\max$ values for $\log (Z)$ were set to be between -0.3 and $0.3 \times$ solar metallicity as a reasonable range given the galactocentric distances of the reddened stars in our sample. For the stellar parameters, $\log \left(T_{\text {eff }}\right), \log (g)$, and $\log (Z)$, the base priors are given by the allowed model space as defined by the TLusty stellar atmosphere grid. For $\log \left(T_{\text {eff }}\right)$, we add an additional multiplicative Gaussian prior based on the literature spectral type (Table 2) and assuming an uncertainty of one subclass in spectral type. We use the Conti et al. (2008) calibration of spectral type to $\log \left(T_{\text {eff }}\right)$. The results of the fitting have been used to calculate uncertainties on all of the fit parameters. These uncertainties include a full account of the sources of noise, including spectral mismatch illustrating one of the strengths of the method.

Since the stars in M31 are being paired with unreddened stellar models, the foreground MW dust extinction must be removed explicitly. Older estimates of the M31 foreground ( $E$ $(B-V) \sim 0.08 \mathrm{mag})$ are discussed in Bianchi et al. (1996). Newer estimates including Schlegel et al. (1998) and Schlafly $\&$ Finkbeiner (2011) give an average $E(B-V)=0.06 \mathrm{mag}$ (Cordiner et al. 2011). This estimate for the MW foreground has been confirmed by a survey of M31 star-forming regions (Bianchi et al. 2012). Thus, an MW foreground extinction component of $E(B-V)=0.06$, assuming $R_{V}=3.1 \mathrm{CCM}$ dust, is included as part of the fitting process described above. Three of the seven "reddened" stars in the sample had small 
Table 1

New and Archival HST/STIS Observations in M31

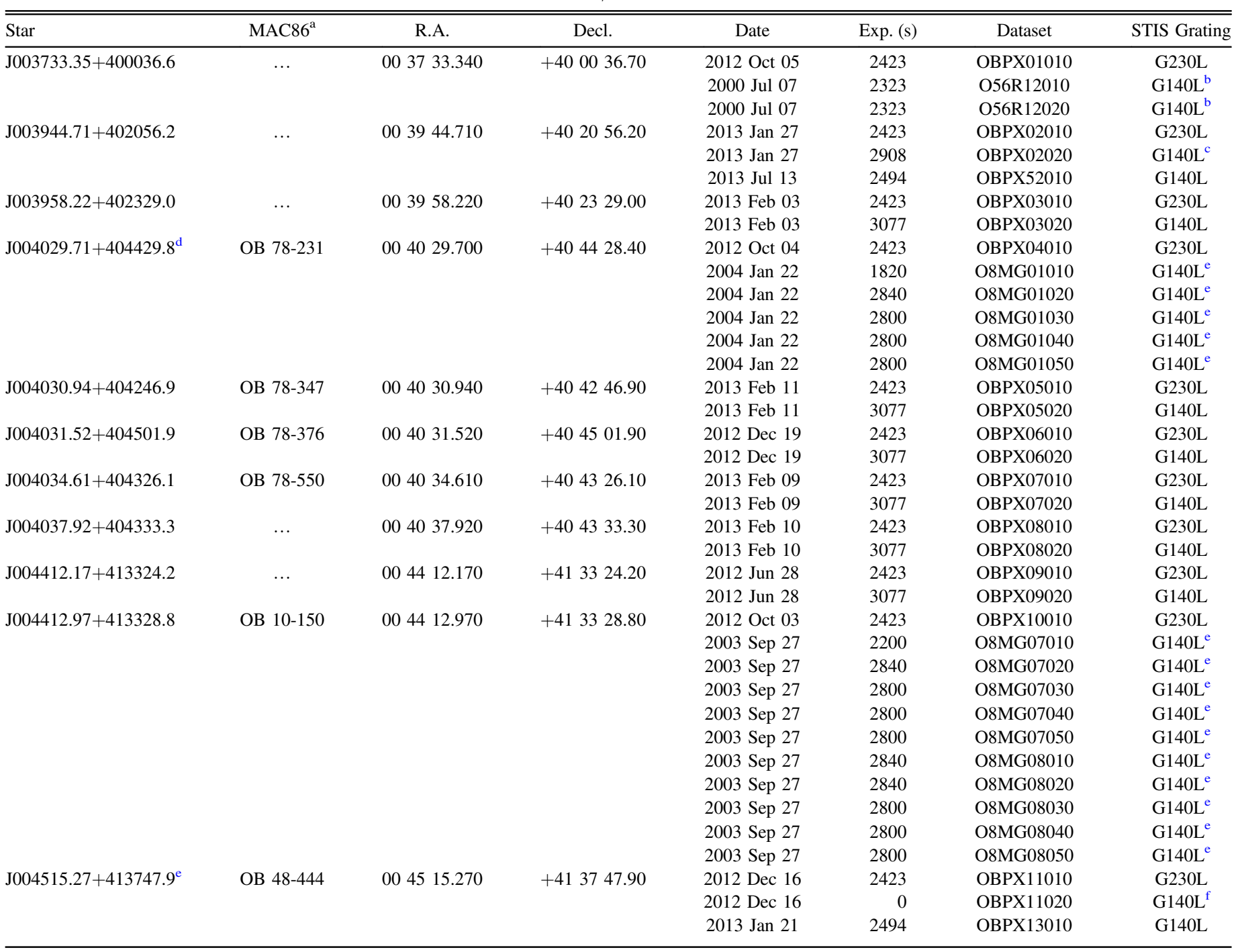

Notes.

a Massey et al. (2006).

b Bresolin et al. (2002), 52" $\times 0$." 2 aperture.

${ }^{c}$ G140L failed. Re-observed on 2013 July 13.

d Target in Bianchi et al. (1996).

e Data obtained for GO 9794.

${ }^{\mathrm{f}}$ G140L failed. Re-observed on 2013 January 21.

$E(B-V)$ values between 0.09 and 0.16 so these curves are not used in the analysis presented here. The positions of the four remaining stars are plotted on a GALEX image of M31 in Figure 1 and their HST/STIS spectra are plotted in Figure 2. The extinction curves for these four significantly reddened sightlines are shown in Figure 3 with a five-point smoothing. The model fit parameter results for each star are given in Tables 4-6. The extinction curves are fit with the Fitzpatrick \& Massa (1990, FM) parameters. The fit parameters and $1 \sigma$ uncertainties are tabulated based on the $17 \%, 50 \%$, and $83 \%$ values of the marginalized $1 \mathrm{D}$ posterior probability distribution functions generated from the EMCEE results. Table 5 gives the best fit and the $50 \%$ (median probability) fit for each extinction curve. We give the dust column results using $E(B-V)$ as this measurement was well behaved (small uncertainties) whereas the measurements of $A(V)$ and $R(V)$ have very large uncertainties. The $c_{1}$ parameter is not fit. We include it here assuming that $c_{1}=2.09-2.84 c_{2}$ (Fitzpatrick \& Massa 2007).

The column density of $\mathrm{H}$ I along each sightline in the sample was estimated by measuring Ly $\alpha$ absorption lines in the $H S T$ / STIS spectra (Diplas \& Savage 1994). This was done simultaneously as part of the model fitting. The estimates for $N\left(\mathrm{H}_{\mathrm{I}}\right)$ are given in Table 6. Also calculated and listed in Table 6 are the gas-to-dust ratios, $N(\mathrm{HI}) / E(B-V)$. There are also $21 \mathrm{~cm}$ observations of $\mathrm{H}$ i for the four sightlines (Cordiner et al. 2011). Unlike the Ly $\alpha$ column densities, which just measure the $\mathrm{H}$ i in front of the star, the $21 \mathrm{~cm}$ column densities are for the entire sightline. Thus the best estimate of the gas-todust ratio is $0.5 N(\mathrm{HI}) / E(B-V)$ for the $21 \mathrm{~cm}$ observations. These are also listed in Table 6 . Because the fraction of the $\mathrm{HI}$ column density that is along the sightline to the star in unknown, the uncertainties are assumed to be $\pm 100 \%$. 
Table 2

Stellar Parameters

\begin{tabular}{|c|c|c|c|c|c|}
\hline Star & $V$ & $B-V$ & Sp.T. & $E(B-V)^{\mathrm{a}}$ & References $^{\mathrm{b}}$ \\
\hline J003733.35+400036.6 & 18.16 & -0.21 & B2 Ia & -0.04 & (2) \\
\hline J003944.71+402056.2 & 18.2 & 0.15 & O9.7 Ib & 0.42 & (1) \\
\hline J003958.22+402329.0 & 18.97 & 0.09 & B0.7 Ia & 0.30 & (1) \\
\hline J004030.94+404246.9 & 18.87 & -0.15 & O9.5 Ib & 0.12 & (1) \\
\hline J004031.52+404501.9 & 18.92 & -0.15 & B0.5 Ia & 0.07 & (1) \\
\hline J004034.61+404326.1 & 18.67 & 0.15 & B1 Ia & 0.34 & (1) \\
\hline $\mathrm{J} 004412.97+413328.8$ & 19.18 & -0.04 & O8.5 Ia(f) & 0.24 & (3) \\
\hline J004515.27+413747.9 & 19.10 & -0.02 & O8 I & 0.26 & (3) \\
\hline
\end{tabular}

Notes.

a Total $E(B-V)$ including MW foreground assuming the measured spectral type and intrinsic colors (Fitzgerald 1970).

b Spectral types are from: (1) Cordiner et al. (2011), (2) Bresolin et al. (2002), and (3) Massey et al. (1995).

Table 3

Model Parameters

\begin{tabular}{|c|c|c|c|}
\hline Parameter & Description & Min & Max \\
\hline \multicolumn{4}{|c|}{ M31 Components } \\
\hline $\log \left(T_{\text {eff }}\right)$ & effective temperature & 4.18 & 4.74 \\
\hline $\log (g)$ & surface gravity & 1.75 & 4.75 \\
\hline $\log (Z)$ & metallicity & -0.3 & 0.3 \\
\hline$A(V)$ & $V$ band extinction & 0.0 & 4.0 \\
\hline$R(V)$ & $A(V) / E(B-V)$ & 1.0 & 7.0 \\
\hline$c_{2}$ & UV slope & -0.5 & 1.5 \\
\hline$c_{3}$ & $2175 \AA$ A bump height & 0.0 & 6.0 \\
\hline$c_{4}$ & FUV curvature & -0.2 & 2.0 \\
\hline$x_{0}$ & $2175 \AA ̊$ bump centroid & 4.55 & 4.65 \\
\hline$\gamma$ & $2175 \AA$ bump width & 0. & 2.5 \\
\hline $\log \left(\mathrm{H}_{\mathrm{I}}\right)$ & $\begin{array}{l}\text { M31 H I column } \\
\text { MW Components }\end{array}$ & 19.0 & 24.0 \\
\hline $\log _{\mathrm{MW}}\left(\mathrm{H}_{\mathrm{I}}\right)$ & MW H I column & 18.0 & 22.0 \\
\hline$E(B-V)_{\mathrm{MW}}$ & MW dust column & \multicolumn{2}{|c|}{0.06} \\
\hline$R(V)_{\mathrm{MW}}$ & MW $A(V) / E(B-V)$ & \multicolumn{2}{|c|}{3.1} \\
\hline$v(\mathrm{MW})$ & velocity $\left(\mathrm{km} \mathrm{s}^{-1}\right)$ & \multicolumn{2}{|c|}{0} \\
\hline
\end{tabular}

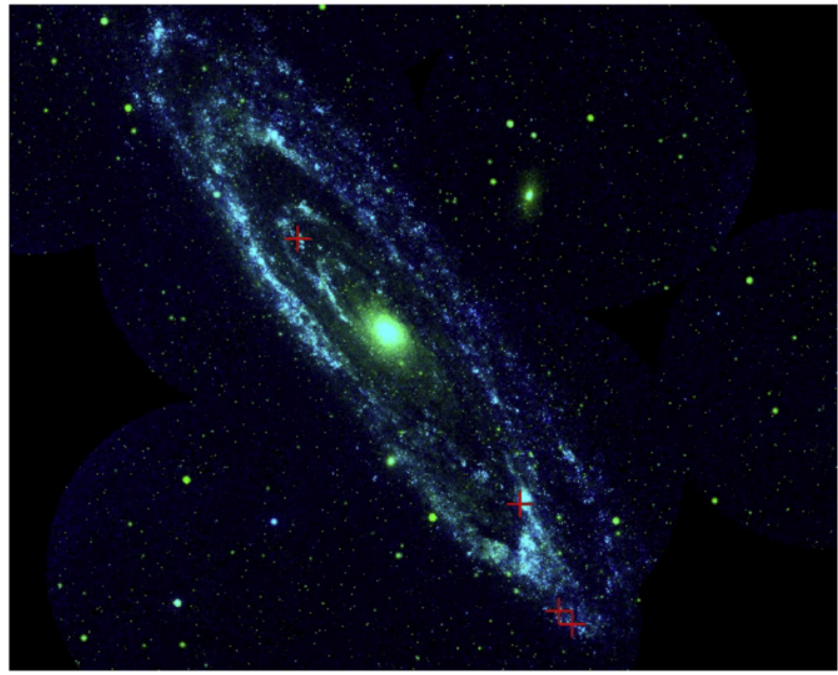

Figure 1. Color GALEX Image of M31 (NUV green, FUV blue) with the locations of the four reddened stars in the sample marked by red crosses.

Table 4

Stellar Parameter Results

\begin{tabular}{|c|c|c|c|c|c|}
\hline \multirow[b]{2}{*}{ Star } & \multicolumn{2}{|c|}{ Prior } & \multicolumn{3}{|c|}{ Fit Parameters } \\
\hline & $\log \left(T_{\text {eff }}\right)$ & $\begin{array}{r}\mathrm{RV} \\
\left(\mathrm{km} \mathrm{s}^{-1}\right)\end{array}$ & $\log \left(T_{\text {eff }}\right)$ & $\log (g)$ & $\log (Z)$ \\
\hline J003944.71+402056.2 & $4.47 \pm 0.03$ & -511 & $4.48_{-0.02}^{+0.03}$ & $2.79_{-0.22}^{+0.21}$ & $0.03_{-0.17}^{+0.16}$ \\
\hline J003958.22+402329.0 & $4.37 \pm 0.04$ & -505 & $4.35_{-0.03}^{+0.04}$ & $2.08_{-0.24}^{+0.52}$ & $0.02_{-0.19}^{+0.19}$ \\
\hline J004034.61+404326.1 & $4.33 \pm 0.02$ & -536 & $4.35_{-0.02}^{+0.02}$ & $2.53_{-0.23}^{+0.47}$ & $0.16_{-0.17}^{+0.09}$ \\
\hline J004412.17+413324.2 & $4.23 \pm 0.04$ & -76 & $4.24_{-0.04}^{+0.04}$ & $2.32_{-0.39}^{+1.11}$ & $0.03_{-0.21}^{+0.18}$ \\
\hline
\end{tabular}

\section{DISCUSSION}

The calculated extinction curves for the four new sightlines in M31 are shown in Figure 3. These curves have been corrected for MW foreground, and thus should reflect the extinction properties of interstellar dust in M31. These curves are a great improvement on the data presented in Figure 5 of Bianchi et al. (1996), which are an average of several lowreddening sightlines. That curve seemed to show a weak bump along with an MW-like FUV extinction.
The new curves sample dust in M31 at a range of galactocentric distances and in different regions of M31 as shown in Figure 1. We are sampling sightlines separated by kiloparsecs, much further apart than any sampled in our own Galaxy. The extinction curve for J003944.71+402056.2 is the only sightline in the sample with an estimated value of $R_{V} \sim 3.3$. This value is consistent with its measured extinction curve shown in Figure 3, which looks very similar to the average MW (CCM $\left.R_{V}=3.1\right)$ extinction curve overplotted in 


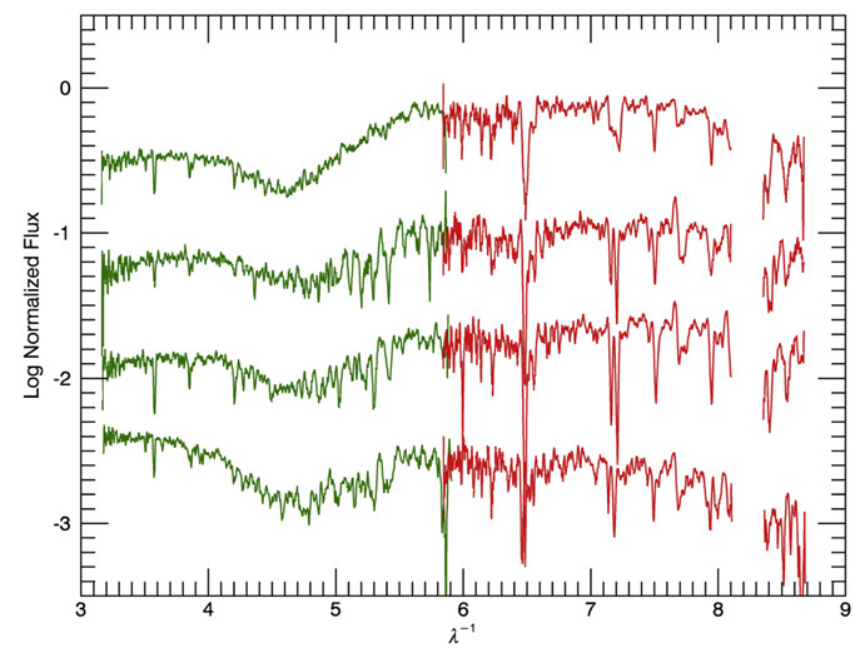

Figure 2. HST/STIS G140L (red) and G230L (green) spectra for four significantly redded stars. From top to bottom, the spectra are J003944.71 +402056.2, J003958.22+402329.0, J004034.61+404326.1, and J004412.17 +413324.2 .

red. This star appears to be associated with an $\mathrm{H}$ II region, and shows strong diffuse interstellar band (DIB) features (Baade \& Arp 1964; Cordiner et al. 2011). J003944.71+402056.2 lies very close in projection to J003958.22+402329.0, but may not be very close in three dimensions. The curve for J003958.22 +402329.0 is significantly different from J003944.71 +402056.2 with a smaller FUV curvature parameter $\left(c_{4}\right)$ and a weaker bump as measured by $c_{3} / \gamma^{2}$ (Fitzpatrick \& Massa 1990). The extinction curve for J004034.61+404326.1 is similar to J003958.22+402329.0 but with a stronger bump. This sightline shows weak DIB features (Cordiner et al. 2011).

The most reddened sightline, J004412.17+413324.2, shows a curve reminiscent of the 30-Dor region of the LMC or an MW CCM $R_{V} \sim 2$ curve (Misselt et al. 1999). However, the lack of FUV curvature $\left(c_{4}\right)$ makes it even flatter than the LMC30-Dor curves. It is also associated with an $\mathrm{H}$ II region (Baade \& Arp 1964; Cordiner et al. 2011). The J004034.61+404326.1 and J003958.22+402329.0 extinction curves show a similar wavelength dependence. This can be seen in Figure 4 where the FM parameters for MW, LMC, SMC, and the new M31 sightlines are plotted against $E(B-V)$. The Spitzer $8 \mu \mathrm{m}$ intensity is relatively strong close to J004412.17+413324.2 possibly indicating the presence of a dense dust cloud (Cordiner et al. 2011). It also shows weak DIB features for its reddening as do sightlines in the LMC (Cox et al. 2006). The UV extinction observed through five dusty clumps in the bulge of M31 has been measured using filter photometry with HST and Swift (Dong et al. 2014). They suggest that the curves are steeper than the average MW extinction, perhaps with $R_{V} \sim 2.5$ but there is a lot of scatter in their curves. Their result
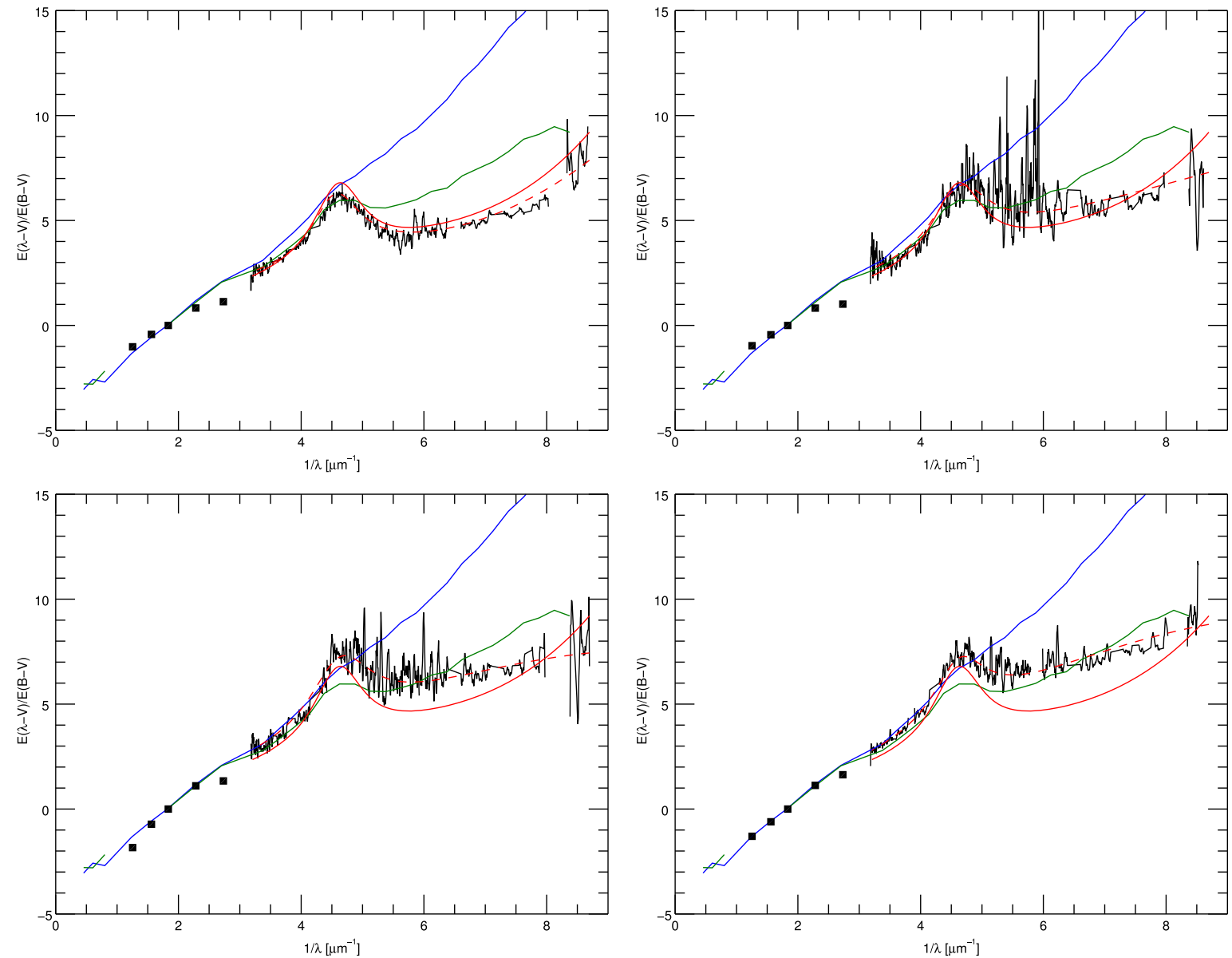

Figure 3. Extinction curves for the target sample using the best fits with atmosphere models. The extinction curves are J003944.71+402056.2 (upper left), J003958.22 +402329.0 (upper right), J004034.61+404326.1 (lower left), and J004412.17+413324.2 (lower right). The black line is the extinction curve using STIS spectra, and the black squares are UBVRI. Shown for comparison are the SMC average extinction curve (blue), the LMC (30-Dor) average extinction curve (green), and the MW CCM $R(V)=3.1$ (red). The best fit to the FM parameters, listed in Table 5, is represented by the red dashed line. 
Table 5

Dust FM Parameter Results ${ }^{\mathrm{a}}$

\begin{tabular}{|c|c|c|c|c|c|c|c|}
\hline Star & $E(B-V)$ & $c_{1}{ }^{\mathrm{b}}$ & $c_{2}$ & $c_{3}$ & $c_{4}$ & $x_{0}$ & $\gamma$ \\
\hline J003944.71+402056.2 & $0.35_{-0.04}^{+0.04}$ & $0.24_{-0.31}^{+0.28}$ & $0.65_{-0.10}^{+0.11}$ & $3.58_{-1.01}^{+1.21}$ & $0.41_{-0.07}^{+0.08}$ & $4.57_{-0.02}^{+0.03}$ & $1.07_{-0.14}^{+0.13}$ \\
\hline & 0.37 & 0.33 & 0.62 & 3.05 & 0.38 & 4.57 & 0.99 \\
\hline J003958.22+402329.0 & $0.25_{-0.03}^{+0.04}$ & $-0.30_{-0.68}^{+0.54}$ & $0.84_{-0.19}^{+0.24}$ & $3.63_{-1.72}^{+1.55}$ & $-0.02_{-0.09}^{+0.09}$ & $4.62_{-0.03}^{+0.02}$ & $1.13_{-0.24}^{+0.18}$ \\
\hline $\mathrm{J} 004034.61+404326.1$ & $\begin{array}{l}0.24 \\
0.26_{-0.03}^{+0.03}\end{array}$ & $\begin{array}{c}-0.41 \\
-0.95_{-0.51}^{+0.45}\end{array}$ & $\begin{array}{l}0.88 \\
1.07_{-0.16}^{+0.18}\end{array}$ & $\begin{array}{l}3.95 \\
4.27_{-1.35}^{+1.16}\end{array}$ & $\begin{array}{c}-0.01 \\
-0.14_{-0.04}^{+0.07}\end{array}$ & $\begin{array}{l}4.65 \\
4.63_{-0.03}^{+0.01}\end{array}$ & $\begin{array}{c}1.15 \\
1.14_{-0.16}^{+0.15}\end{array}$ \\
\hline $\mathrm{J} 004412.17+413324.2$ & $\begin{array}{l}0.25 \\
0.38_{-0.04}^{+0.05}\end{array}$ & $\begin{array}{c}-0.86 \\
-1.54_{-043}^{+0.51}\end{array}$ & $\begin{array}{l}1.04 \\
1.28_{-018}^{+0.15}\end{array}$ & $\begin{array}{l}5.16 \\
3.93_{-144}^{+1.37}\end{array}$ & $\begin{array}{c}-0.16 \\
0.04_{-016}^{+0.23}\end{array}$ & $\begin{array}{l}4.65 \\
4.61_{-0.04}^{+0.03}\end{array}$ & $\begin{array}{c}1.25 \\
1.17_{-025}^{+0.22}\end{array}$ \\
\hline
\end{tabular}

Notes.

${ }^{a}$ For each star, the first line is the $50 \%$ (median probability) fit and the second line is the best fit.

$\mathrm{b}$ The $c_{1}$ parameter is not fit. It is assumed that $c_{1}=2.09-2.84 c_{2}$ (Fitzpatrick \& Massa 2007).

Table 6

Gas-to-dust Ratio Measurements

\begin{tabular}{|c|c|c|c|c|c|c|}
\hline & \multirow[b]{2}{*}{ GC Dist. ${ }^{a}$} & \multicolumn{3}{|c|}{ Fit Results } & \multicolumn{2}{|c|}{$21 \mathrm{~cm}$ Results } \\
\hline Star & & $E(B-V)$ & $N\left(\mathrm{H} \mathrm{I}^{\mathrm{b}}{ }^{\mathrm{b}}\right.$ & $N\left(\mathrm{H}_{\mathrm{I}}\right) / E(B-V)^{\mathrm{c}}$ & $N(\mathrm{H} \mathrm{I})^{\mathrm{d}}$ & $N(\mathrm{H} \mathrm{I}) / E(B-V)^{\mathrm{e}}$ \\
\hline $\mathrm{J} 003944.71+402056.2$ & 14.0 & $0.35_{-0.04}^{+0.04}$ & $4.2_{-2.0}^{+1.7}$ & $11.5_{-5.6}^{+5.1}$ & 4.5 & 6.4 \\
\hline J004034.61+404326.1 & 8.8 & $0.26_{-0.03}^{+0.03}$ & $6.2_{-2.0}^{+1.6}$ & $23.7_{-7.8}^{+6.9}$ & 2.3 & 4.4 \\
\hline J004412.17+413324.2 & 5.2 & $0.38_{-0.04}^{+0.05}$ & $<2.7$ & $<7.2$ & 2.0 & 2.5 \\
\hline
\end{tabular}

Notes.

${ }^{\text {a }}$ Projected galactocentric distance from the center of M31 in kpc.

${ }^{\mathrm{b}} N\left(\mathrm{H}_{\mathrm{I}}\right)$ from Ly $\alpha$ absorption. In units of $10^{21}$ atoms $\mathrm{cm}^{-2}$.

${ }^{c}$ In units of $10^{21}$ atoms $\mathrm{cm}^{-2} \mathrm{mag}^{-1}$.

${ }^{\mathrm{d}} N\left(\mathrm{H}_{\mathrm{I}}\right)$ from $21 \mathrm{~cm}$ observations. In units of $10^{21}$ atoms $\mathrm{cm}^{-2}$ (Cordiner et al. 2011).

e $0.5 N(\mathrm{H} \mathrm{I}) / E(B-V)$. In units of $10^{21}$ atoms $\mathrm{cm}^{-2} \mathrm{mag}^{-1}$.
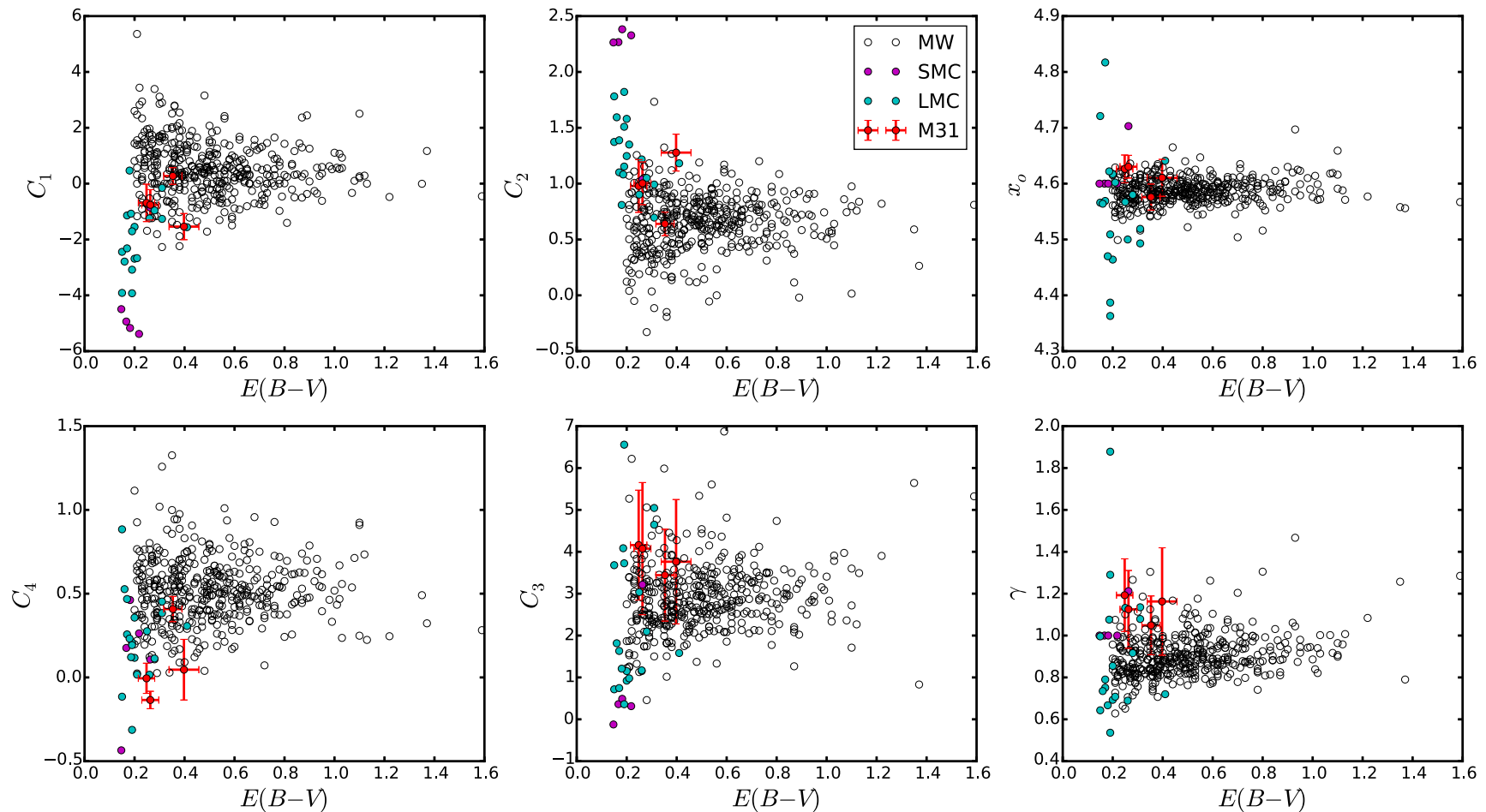

Figure 4. FM parameters plotted against $E(B-V)$ for sightlines in the MW, LMC, SMC, as well as the new sightlines in M31 presented here (Gordon et al. 2003 ; Valencic et al. 2004). 

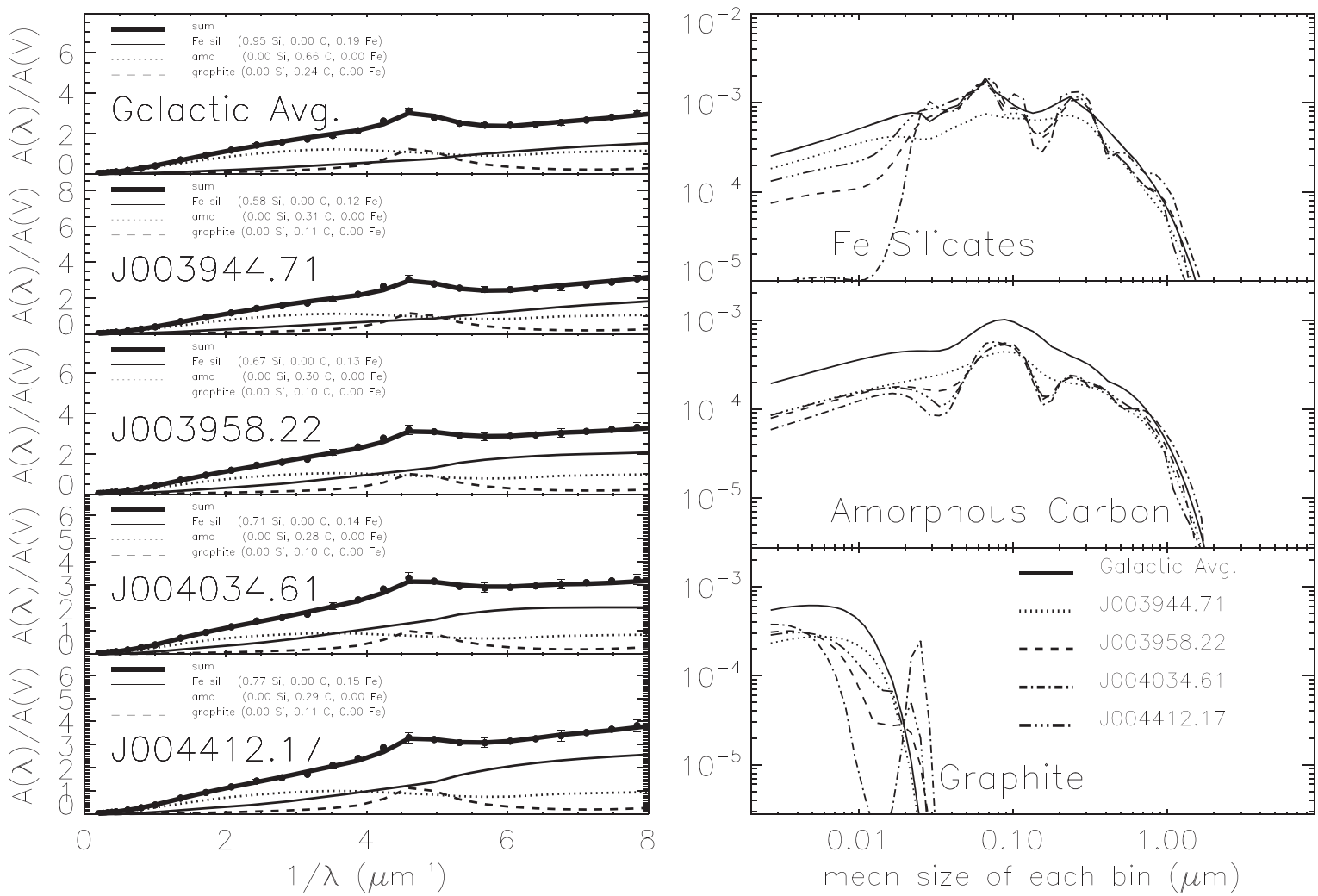

Figure 5. Left: three-component extinction models for the four M31 sightlines along with the average MW extinction for comparison. Each panel shows the model fit to the extinction curve, including the contribution of each component. The fraction of the available $\mathrm{Si}, \mathrm{Fe}$, and $\mathrm{C}$ (amorphous carbon and graphite) utilized is listed in the figure legend. Right: three-component extinction models for the same sightlines. Each panel contains the resulting mass distributions relative to the mass of hydrogen.

supports that of Melchior et al. (2000) which implied $R_{V} \sim 2.1$ using only BVRI photometry for one sightline in the bulge of M31. The extinction curve for J004412.17+413324.2, which is the closest of the stars in our sample to the M31 bulge, at a projected galactocentric distance of $5 \mathrm{kpc}$, resembles an MW curve with an $R_{V} \sim 2$ but is closer to an LMC 30-Dor curve. These extinction measurements all show evidence for low $R_{V}$, and small average grain sizes in the highest metallicity regions of M31.

The gas-to-dust ratios measured here using Ly $\alpha$ absorption lines for J003944.71+402056.2, J003958.22+402329.0, and J004034.61+404326.1 may be higher than the average MW value $\left(5.8 \times 10^{21}\right.$ atoms $\left.\mathrm{cm}^{-2} \mathrm{mag}^{-1}\right)$ (Bohlin et al. 1978), though these values are quite uncertain. The projected galactocentric distance from the center of M31 for these stars is between 8.8 and $14.0 \mathrm{kpc}$ where the metallicity is approximately solar (Sanders et al. 2012). J004412.17 +413324.2 , the most reddened star, has the lowest $N\left(\mathrm{H}_{\mathrm{I}}\right)$ column density and therefore the lowest gas-to-dust ratio in the sample, significantly lower than the average MW value. It is at a projected galactocentric distance of only $5.2 \mathrm{kpc}$, which corresponds to a metallicity of about 1.6 solar, thus a low gasto-dust ratio would be expected (Sanders et al. 2012). Unfortunately, the measured $\mathrm{Ly} \alpha N\left(\mathrm{H}_{\mathrm{I}}\right)$ is very uncertain for this sightline. However, the $21 \mathrm{~cm}$ data for the same sightline, listed in Table 6, also indicate that the gas-to-dust ratio is low. The $21 \mathrm{~cm} N\left(\mathrm{H}_{\mathrm{I}}\right)$ estimates for the four stars imply lower gas-todust ratios than the Ly $\alpha$ estimates, more in line with the average MW value, but since the fraction of the $N(\mathrm{HI})$ along the line of sight to each star is uncertain, the column densities could be higher or even lower. (Cordiner et al. 2011). Taking an average of the four sightlines using the $21 \mathrm{~cm}$ data, we get a gas-to-dust ratio of $5.3 \times 10^{21}$ atoms cm $\mathrm{cm}^{-2} \mathrm{mag}^{-1}$, close to the MW value.

The measured extinction curves for the four M31 sightlines have been used as inputs for the Maximum Entropy Method (MEM) modeling of the underlying dust grain populations. This analysis is similar to that applied previously to extinction curves in the MW and the Magellanic clouds (Clayton et al. 2003). We use a version of the MEM extinction-fitting algorithm similar to that developed by Kim et al. (1994). MEM uses the mass distribution in which $m(a) d a$ is the mass of dust grains per $\mathrm{H}$ atom in the size interval from $a$ to $a+d a$ rather than using the number of grains as a constraint. A Mathis et al. (1977; MRN-type) model becomes $m(a) \propto a^{-0.5}$. The total mass of dust is constrained using both the gas-to-dust ratio and the available abundances of iron, carbon, and silicon. The fraction of the available silicon and carbon used in the MEM fits covers a very wide range. For example, the MW, which has a relatively low gas-to-dust ratio and high metal abundances, also requires almost $100 \%$ of the available silicon and and $80 \%$ of the carbon (Clayton et al. 2003). The LMC (30-Dor) and the SMC Bar regions both have low metal abundances and high gas-to-dust ratios. Both use $50 \%$ or less of the available silicon and $60 \%-80 \%$ of the carbon. Three general factors determine the fraction of silicon and carbon that any individual sightline will use. First, the higher the gas-to-dust ratio is, the more metals are available in the gas phase. Second, the higher the abundances of metals are, the more material is available to make grains. Finally, high values of the ratio of total-to- 
selective extinction, $R_{V}$, imply a greater than average mass fraction in larger grains.

The MEM fit results are shown in Figure 5. The fits were made using three interstellar dust components, Fe-silicate, graphite, and amorphous carbon (Jaeger et al. 1994; Dorschner et al. 1995; Clayton et al. 2003). We have adopted the following MW abundances for the MEM fitting, $\mathrm{Si} / \mathrm{H}=4.0 \times 10^{-5}$, $\mathrm{C} / \mathrm{H}=3.20 \times 10^{-4}$, and $\mathrm{Fe} / \mathrm{H}=4.1 \times 10^{-5}$ (Clayton et al. 2003). As described above, the measured gas-to-dust ratios may be higher than the MW for three of the sightlines and lower for J004412.17+413324.2 but have large uncertainties. Also, based on their projected positions in M31, the abundances should be near solar for J003944.71+402056.2, J003958.22+402329.0, and J004034.61+404326.1 and super-solar for J004412.17 +413324.2 . The latter sightline has the lowest gas-to-dust ratio as expected. Therefore, three MEM fitting runs were performed for the four M31 sightlines, assuming solar abundances along with three different gas-to-dust ratios, MW $\left(5.8 \times 10^{21}\right.$ atoms cm $\left.\mathrm{cmg}^{-1}\right), 1 / 2 \times \mathrm{MW}$, and $2 \times \mathrm{MW}$. The results for the run with solar abundances and the $2 \times \mathrm{MW}$ gas-to-dust ratio are shown in Figure 5. Also shown in Figure 5 are the values for the MW CCM $R_{V}=3.1$ average extinction curve.

Of the three MEM runs, in the first two, with solar abundances, and MW and $1 / 2 \times \mathrm{MW}$ gas-to-dust ratios, there is not enough $\mathrm{Si}$ and $\mathrm{C}$ available to make the dust grains required to fit the observed extinction. In the last case, shown in Figure 5, the available abundances are enough to make the grains needed. The four M31 sightlines modeled here use $53 \%-74 \%$ of the $\mathrm{Si}, 11 \%-15 \%$ of the $\mathrm{Fe}, 13 \%-16 \%$ of the graphite, and $38 \%-41 \%$ of the amorphous carbon available. The results show that the M31 extinction curves can only be fit where the abundances are greater than solar, the gas-todust ratio is greater than the average MW ratio or a combination of both. The large measured gas-to-dust ratios for J003944.71+402056.2, J003958.22+402329.0, and $\mathrm{J} 004034.61+404326.1$ and solar abundances are needed to fit those sightlines. The most reddened sightline, J004412.17 +413324.2 , which lies closest to the center of M31 is problematic. The abundances for this sightline would need to be very super-solar if the small measured gas-to-dust ratio is correct. Unfortunately, there is not much information on $R_{V}$ for these sightlines, only J003944.71+402056.2 with $R_{V}=3.3$ has an estimate, which seems reasonable for its observed extinction curve.

\section{SUMMARY}

The new UV extinction curves presented here provide a tantalizing glimpse into the interstellar dust properties in M31. Unlike the MW, the dust in M31 can be sampled across the whole galactic disk. These results are a significant improvement on previous M31 extinction curves (Bianchi et al. 1996). The sample is small but the extinction properties range from a curve very similar to CCM $R_{V}=3.1$ seen toward J003944.71 +402056.2 which is at a projected distance from the galactic center of $14 \mathrm{kpc}$ to a curve similar to LMC (30-Dor) extinction seen toward $\mathrm{J} 004412.17+413324.2$ which is at a projected distance of $5 \mathrm{kpc}$.

The new extinction curves show similarities to those seen in the MW and the LMC. The highest metallicity sightline, which is closest to the M31 bulge, and has the lowest gas-to-dust ratio shows an extinction curve consistent with a low value of $R_{V}$ or possibly with the LMC 30-Dor region. Many more sightlines need to be studied to map out the extinction properties of M31 and to investigate the link between UV extinction properties and global characteristics such as metallicity and star formation activity.

This study was supported by grant HST-GO-12562.01 from NASA/STScI. Some of the data presented in this paper were obtained from the Mikulski Archive for Space Telescopes (MAST). STScI is operated by the Association of Universities for Research in Astronomy, Inc., under NASA contract NAS526555. We would like to thank Jonathan Sick for providing JK photometry for this study.

\section{REFERENCES}

Aiello, S., Barsella, B., Chlewicki, G., et al. 1988, A\&AS, 73, 195

Baade, W., \& Arp, H. 1964, ApJ, 139, 1027

Bianchi, L., Clayton, G. C., Bohlin, R. C., Hutchings, J. B., \& Massey, P. 1996, ApJ, 471, 203

Bianchi, L., Efremova, B., Hodge, P., \& Kang, Y. 2012, AJ, 144, 142

Bohlin, R. C., Savage, B. D., \& Drake, J. F. 1978, ApJ, 224, 132

Bonanos, A. Z., Stanek, K. Z., Sasselov, D. D., et al. 2003, AJ, 126, 175

Bresolin, F., Kudritzki, R.-P., Lennon, D. J., et al. 2002, ApJ, 580, 213

Calzetti, D., Kinney, A. L., \& Storchi-Bergmann, T. 1994, ApJ, 429, 582

Cardelli, J. A., Clayton, G. C., \& Mathis, J. S. 1989, ApJ, 345, 245

Cardelli, J. A., Sembach, K. R., \& Mathis, J. S. 1992, AJ, 104, 1916

Clayton, G. C., Gordon, K. D., \& Wolff, M. J. 2000, ApJS, 129, 147

Clayton, G. C., \& Martin, P. G. 1985, ApJ, 288, 558

Clayton, G. C., Wolff, M. J., Gordon, K. D., et al. 2004, AJ, 127, 3382

Clayton, G. C., Wolff, M. J., Sofia, U. J., Gordon, K. D., \& Misselt, K. A. 2003, ApJ, 588, 871

Conti, P. S., Crowther, P. A., \& Leitherer, C. 2008, From Luminous Hot Stars to Starburst Galaxies (Cambridge: Cambridge Univ. Press)

Cordiner, M. A., Cox, N. L. J., Evans, C. J., et al. 2011, ApJ, 726, 39

Cox, A. N. 2000, in Allen's Astrophysical Quantities, ed. A. N. Cox (4th ed.; New York: AIP Press)

Cox, N. L. J., Cordiner, M. A., Cami, J., et al. 2006, A\&A, 447, 991

De Marchi, G., \& Panagia, N. 2014, MNRAS, 445, 93

Diplas, A., \& Savage, B. D. 1994, ApJ, 427, 274

Dong, H., Li, Z., Wang, Q. D., et al. 2014, ApJ, 785, 136

Dorschner, J., Begemann, B., Henning, T., Jaeger, C., \& Mutschke, H. 1995 A\&A, 300, 503

Drout, M. R., Massey, P., Meynet, G., Tokarz, S., \& Caldwell, N. 2009, ApJ, 703, 441

Fitzgerald, M. P. 1970, A\&A, 4, 234

Fitzpatrick, E. L. 1985, ApJ, 299, 219

Fitzpatrick, E. L. 1999, PASP, 111, 63

Fitzpatrick, E. L., \& Massa, D. 1990, ApJS, 72, 163

Fitzpatrick, E. L., \& Massa, D. 2005, AJ, 130, 1127

Fitzpatrick, E. L., \& Massa, D. 2007, ApJ, 663, 320

Foreman-Mackey, D., Hogg, D. W., Lang, D., \& Goodman, J. 2013, PASP, 125,306

Gordon, K. D., Calzetti, D., \& Witt, A. N. 1997, ApJ, 487, 625

Gordon, K. D., \& Clayton, G. C. 1998, ApJ, 500, 816

Gordon, K. D., Clayton, G. C., Misselt, K. A., Landolt, A. U., \& Wolff, M. J. 2003, ApJ, 594, 279

Gordon, K. D., Hanson, M. M., Clayton, G. C., Rieke, G. H., \& Misselt, K. A. 1999, ApJ, 519, 165

Gordon, K. D., et al. 2015, ApJ, in preparation

Jaeger, C., Mutschke, H., Begemann, B., Dorschner, J., \& Henning, T. 1994, A\&A, 292, 641

Kim, S.-H., Martin, P. G., \& Hendry, P. D. 1994, ApJ, 422, 164

Lanz, T., \& Hubeny, I. 2003, ApJS, 146, 417

Lanz, T., \& Hubeny, I. 2007, ApJS, 169, 83

Lequeux, J., Maurice, E., Prevot-Burnichon, M.-L., Prevot, L., \& Rocca-Volmerange, B. 1982, A\&A, 113, L15

Maíz Apellániz, J., \& Rubio, M. 2012, A\&A, 541, A54

Massa, D., Savage, B. D., \& Fitzpatrick, E. L. 1983, ApJ, 266, 662

Massey, P., Armandroff, T. E., Pyke, R., Patel, K., \& Wilson, C. D. 1995, AJ, 110,2715

Massey, P., Olsen, K. A. G., Hodge, P. W., et al. 2006, AJ, 131, 2478

Mathis, J. S., Rumpl, W., \& Nordsieck, K. H. 1977, ApJ, 217, 425 
Melchior, A.-L., Viallefond, F., Guélin, M., \& Neininger, N. 2000, MNRAS, 312, L29

Misselt, K. A., Clayton, G. C., \& Gordon, K. D. 1999, ApJ, 515, 128

Prevot, M. L., Lequeux, J., Prevot, L., Maurice, E., \& Rocca-Volmerange, B. 1984, A\&A, 132, 389

Riess, A. G., Fliri, J., \& Valls-Gabaud, D. 2012, ApJ, 745, 156

Rubin, V. C., \& Ford, W. K., Jr. 1970, ApJ, 159, 379

Sanders, N. E., Caldwell, N., McDowell, J., \& Harding, P. 2012, ApJ, 758, 133

Schlafly, E. F., \& Finkbeiner, D. P. 2011, ApJ, 737, 103
Schlegel, D. J., Finkbeiner, D. P., \& Davis, M. 1998, ApJ, 500, 525

Sick, J., Courteau, S., Cuillandre, J.-C., et al. 2014, AJ, 147, 109

Smartt, S. J., Crowther, P. A., Dufton, P. L., et al. 2001, MNRAS, 325, 257

Valencic, L. A., Clayton, G. C., \& Gordon, K. D. 2004, ApJ, 616, 912

Venn, K. A., McCarthy, J. K., Lennon, D. J., et al. 2000, ApJ, 541, 610

Whittet, D. C. B., \& van Breda, I. G. 1978, A\&A, 66, 57

Witt, A. N., Bohlin, R. C., \& Stecher, T. P. 1984, ApJ, 279, 698

Zaritsky, D., Kennicutt, R. C., Jr., \& Huchra, J. P. 1994, ApJ, 420, 87 